\title{
Atomically Resolved 3D Shape Determination of a MgO Crystal Using a Single Aberration Corrected HRTEM Image
}

\author{
Chun-Lin Jia ${ }^{1,2,3}$, Shao-Bo Mi ${ }^{1,4}$, Juri Barthel ${ }^{3,5}$, Dawei Wang ${ }^{1}$, Rafal E. Dunin-Borkowski ${ }^{2,3}$, Knut W. \\ $\mathrm{Urban}^{2,3}$, Andreas Thust ${ }^{2,3}$ \\ ${ }^{1}$ International Center of Dielectric Research, The School of Electronic and Information Engineering, \\ Xi'an Jiaotong University, Xi'an 710049, China. \\ ${ }^{2}$ Peter Grünberg Institute, Forschungszentrum Jülich GmbH, 52425 Jülich, Germany. \\ ${ }^{3}$ Ernst Ruska-Centre for Microscopy and Spectroscopy with Electrons, Forschungszentrum Jülich \\ $\mathrm{GmbH}, 52425$ Jülich, Germany. \\ ${ }^{4}$ Shenyang National Laboratory for Materials Science, Institute of Metal Research, Chinese Academy of \\ Sciences, Shenyang 110016, China. \\ ${ }^{5}$ Central Facility for Electron Microscopy, RWTH Aachen University, Ahornstr. 55, 52074 Aachen, \\ Germany.
}

The introduction of aberration correction to high-resolution electron microscopy (HRTEM) almost two decades ago facilitated the access to atomic structure information in a revolutionary way and enabled numerous unprecedented materials science results [1]. However, the vast majority of structure characterizations obtained by TEM, regardless whether the coherent TEM technique or the alternative scanning technique (STEM) were applied, were restricted to the determination of the $x$ - and $y$-coordinates of crystalline atom columns in the plane perpendicular to the incident electron beam. Due to the fact that the depth resolution of the TEM technique along the beam direction is inferior to its lateral resolution, full 3D structure determinations on an atomic level remain extremely challenging. A general strategy to solve the $3 \mathrm{D}$ problem is the use of tomographic approaches, where a multitude of images is taken from different observation directions. Such multi-image approaches are highly demanding in the atomic resolution regime due to the need to record and evaluate a large number of images in a consistent way, and due to potential radiation damage problems. Alternatively, single-image approaches have been used for crystalline objects, where only one exposure is taken along a crystallographic zone axis. So far, single-image approaches were restricted to counting the number of atoms contained in atomic columns. However, a full 3D determination of the crystal shape would additionally require a sufficiently accurate determination of the z-position of the lower and upper column endpoints, which has not been achieved so far with the single-image approach.

In the present work we exploit the full depth resolution present in a single aberration corrected HRTEM image by abandoning the hitherto frequently made simplification of a single global defocus value, which corresponds to an idealized flat object exit surface. We demonstrate that the full 3D information about the shape of a $\mathrm{MgO}$ crystal can be retrieved in a unique way when extending the atom counting capabilities of the HRTEM technique by the concept of atomically corrugated object surfaces. By determining the exact number of atoms in each individual crystal column, and by determining now in addition also the z-positions of the column endpoints, the complete crystal shape can be retrieved.

Our 3D determination of the crystal shape is based on refining an atomic structure model in such a way that a HRTEM image simulated on the basis of this model fits best to the experimental image. The simulation parameters include object data (structure model to be refined, Debye-Waller factors (DWF), absorption parameter), as well as microscope parameters (optical aberrations, information limit, camera 
modulation transfer function (MTF), image spread due to thermal magnetic field noise [2]). A part of these parameters is locally refined for each atom column separately (structure model), another part is known from literature (DWF), a further part is known from independent measurements (MTF, information limit), or is globally refined over a large object area sufficiently far away from the position of interest (optical aberrations, image spread, absorption) [3]. The comparison between simulation and experiment is made on the basis of absolute image intensity values [4].

A key component of our atomic-scale 3D structure retrieval procedure is an extended statistical test on the uniqueness of the refined structure model. For this purpose many thousands of images covering all potentially occurring column configurations are simulated on the basis of the globally determined electron optical parameters. After adding realistic noise to these images, the resulting ensemble is used as a pseudo-experimental input for testing the reliability of the previously applied best-fit refinement. Since the correct solution is known in this case, quantitative success rates for the recognition of each individual column configuration can be deduced. The main outcome of this confidence check is that under the given experimental conditions (i) the exact number of atoms in a column can be always determined, and (ii) the z-position of a column can be determined for all columns containing more than two atoms, which allows to perform a nearly unambiguous 3D shape retrieval in our case.

\section{References:}

[1] K.W. Urban, Science 321 (2008) p. 506.

[2] S. Uhlemann et al, Phys. Rev. Lett. 111 (2013) 046101.

[3] C.-L. Jia et al, Microsc. Microanal. 19 (2013) p. 310.

[4] A. Thust, Phys. Rev. Lett. 102 (2009) 220801.
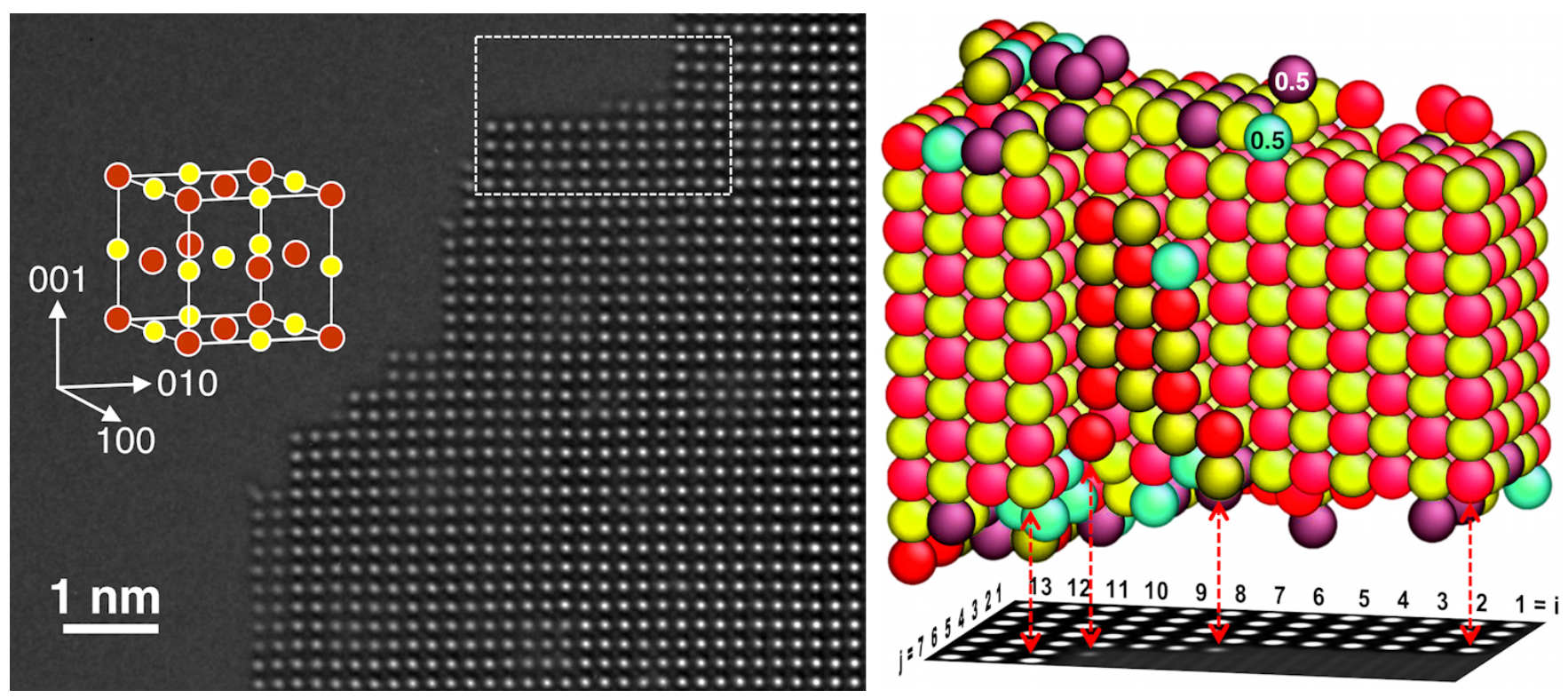

Figure 1. Left: High-resolution image of the edge of a $\mathrm{MgO}$ crystal taken along the [001] zone axis with a CS-corrected FEI Titan 80-300 electron microscope at $300 \mathrm{kV}$ accelerating voltage. The 3D shape reconstruction was performed at the area indicated by the dashed box. Right: 3D structure model retrieved from the boxed area on the left. Red balls indicate $\mathrm{Mg}$ atoms, yellow balls $\mathrm{O}$ atoms, purple balls indicate formally half-occupied $\mathrm{Mg}$ positions, and green balls formally half-occupied $\mathrm{O}$ positions. 